\title{
Using the RE-AIM framework to identify and describe nutritional feeding styles and intervention model best practices for primary caregivers in Africa: A narrative review
}

Nutrition and Health 2021, Vol. 27(2) I7I-180

(C) The Author(s) 2020 Article reuse guidelines: sagepub.com/journals-permissions DOI: 10.1177/0260106020977053 journals.sagepub.com/home/nah (A) SAGE

\author{
Melissa Brown (1) and Nicolette V Roman
}

\begin{abstract}
Background: Current research shows that across the world people are eating poorly. This is leading to increased incidences of nutrition-related health problems. Aim: This paper aims to provide a synthesis of research on the nutritional feeding programs and nutritional models used by primary caregivers in Africa, in order to identify best practice models, programs, and processes from the field of nutritional and feeding intervention development. Methods: The research used a narrative review methodology. The Reach, Effectiveness, Adoption, Implementation and Maintenance (RE-AIM) framework was used to disseminate results to allow for cross-comparison of core components inherent in health promotion interventions. We chose the RE-AIM framework as it facilitates the development, delivery, and evaluation of health interventions. Results: After screening a total of 8220 articles, four studies were deemed relevant for the purposes of this review. The selected studies were the only ones that discussed nutrition interventions or programs with a very clear aim and purpose, even though they did not include any information on implementation, review or evaluation of these interventions/program. No studies focusing on the African context were deemed relevant as none of them focused on best practice models for nutrition education interventions or programs. Conclusions: Of a review of over 8220 articles, four studies were found that discuss nutritional feeding programs and nutritional models used by primary caregivers. Of these four, only one focused on enablers, barriers, and resources, all of which are essential for engaging in health behavior change. And only one focused on sustainability of the interventions.
\end{abstract}

\section{Keywords}

Nutrition program, feeding, parenting, parent feeding models, feeding interventions

\section{Introduction}

Interest in the role that primary caregivers play in child food preferences, nutrition, and weight status has increased in recent years specifically due to the increase of childhood obesity and related conditions. Being overweight affects the social life, physiological state, and psychological health of the child in the long term (Cirak, 2018). Nutrition-related health problems associated with obesity and overweight such as hypertension, type 2 diabetes, dyslipidemia, insulin resistance, sleep apnea, asthma, and non-alcoholic liver fatigue used to occur only in adults. However, these nutrition-related health problems are now common in children due to obesity (Salk et al., 2017). These children also face psychological problems such as depression, anxiety, lack of self-confidence, and sometimes eating disorders. Obesity has a greater association with many chronic diseases than hunger, poverty, smoking, and alcohol use (Hong and Peltzer, 2017). Obesity related to many unhealthy dietary behaviors (consumption of soft drinks, fast food, sweets and snacks, skipping breakfast, and caffeine) has been associated with unhappiness, perceived stress, mental or psychological distress, depression, and poorer sleep (Hayward et al., 2016).

Nutritional data has increased in both quality and quantity, therefore enabling us to develop a better understanding of what people are eating, as well as why what they eat

Child and Family Studies, Social Work Department, University of the Western Cape, Bellville, South Africa

\section{Corresponding author:}

Melissa Brown, Child and Family Studies, Social Work Department, University of the Western Cape, Private Bag XI7, Bellville, South Africa. Email: melissajudithbrown@gmail.com 
matters. There has been a surge in efforts to collect, collate, and analyze data on diets, thereby improving our understanding of what the world eats (Bhutta, 2017). Diets in all countries and wealth groups pose a significant threat to achieving nutrition targets (Bhutta, 2017). Data shows that the world is eating poorly regardless of factors such as wealth or current stage in life. School-aged children, adolescents, and adults are eating too many refined grains and sugary foods and drinks, and not enough foods that promote health such as fruits, vegetables, legumes, and whole grains. About a third (30.3\%) of school-aged children do not eat any fruit daily, yet $43.7 \%$ consume soda every day (Development Initiatives, 2018). Research examining knowledge and use of national dietary guidelines suggests that parents have a good understanding of what they should be feeding their children (Cluss et al., 2013). However, dietary intake and diet quality are difficult to measure. Golan and Weizman (2001) state that a review of prevention interventions for weight-related problems in children concluded that effective interventions should be approached from a health-centered rather than a weight-centered perspective, with the parents as central agents of change.

Despite some limitations, due to their accuracy, Food Frequency Questionnaires (FFQs) are still considered the most efficient and feasible method to assess usual dietary intake (Romanos-Nanclares et al., 2018). This is due to the fact that they are more cost effective than large scale 24hour dietary recalls, due to time and literacy on the part of participants (Moghames et al., 2016). Accuracy of dietary intake information generated by a FFQ is however dependent on the validity and reproducibility of the FFQ in the population it is intended to be used for (Moghames et al., 2016).

Primary caregivers and parents are important agents through which food preferences and intake patterns are set, through both direct and indirect influences. This includes controlling the child's intake, to passively modeling a healthy or unhealthy diet (Shloim et al., 2015). Parental nutrition knowledge and attitudes have been described as important factors for children's healthy food knowledge (Romanos-Nanclares et al., 2018). Research has shown a strong correlation between parental food preferences and their child's food preferences, particularly with fruits, vegetables, sweetened beverages, and meats (Galloway et al., 2006; Yavorsky et al., 2015). Food parenting practices (FPPs) are defined as active techniques or behaviors used by parents to influence a child's food intake (Patel et al., 2018). FPPs have been found to be one of the environmental factors associated with the development of overweight and obesity in childhood and encompass the behaviors used by parents to influence their child's behaviors, attitudes, or beliefs around food and eating. Parents determine what foods are offered to their children and provide an atmosphere in which children are eating (Galloway et al., 2006). Dietary habits established in youth have been shown to track throughout the lifespan (Craigie et al., 2011; Kelder et al., 1994), suggesting that youth dietary interventions should be pursued as a strategy to improve diet, and thereby combat obesity and decrease the risk of developing cancer, cardiovascular disease, and other chronic diseases (Schlechter et al., 2016). In a previous systematic review, Hingle et al. (2010) determined that parental involvement increased dietary intervention effectiveness and also determined which types of parent intervention strategies were most effective. Hingle et al. (2010) were limited in evaluating the effectiveness of parental involvement due to the low quality of reporting, and the small number of studies that evaluated the comparative effectiveness of interventions with and without parental intervention components. In developing an intervention, the ultimate aim is to disrupt or change a behavior or condition, such as increasing parenting skills, promoting connectedness, or promoting strengths and resilience (Walsh, 2003).

In sub-Sahara Africa child feeding strategies that focus on improving feeding practices lack relevance and applicability to caregivers' everyday life, and therefore do not produce the needed change (Ahishakiye et al., 2019). This can be the result of underlying determinants of nutrition which can include poverty, food insecurity, and poor access to health care (Sanders and Reynolds, 2017).

Referring to models, we also refer to the processes and practices used in primary caregivers' nutritional and feeding intervention development as described in the articles found in the searches. This review paper aims to synthesize the research evidence to identify and describe best practice models, programs, or processes in primary caregivers' nutritional and feeding intervention development in Africa.

\section{Methods}

\section{Research approach}

A narrative literature review was employed as they provide useful summaries, in-depth analysis of a specific topic, and may describe the evolution of the subject over time (Mills et al., 2016). Narrative reviews are a discussion of important topics on a theoretical point of view (Jahan et al., 2016). There are three types of narrative reviews of the literature: editorials, commentaries, and overview articles. Narrative overviews, also known as an unsystematic narrative review, are comprehensive narrative syntheses of previously published information (Green et al., 2006). Commonly, these are distinguished from a systematic review in which all primary evidence that meets clear inclusion criteria is retrieved and its quality appraised using explicit and reproducible methodology. Narrative reviews do not always make clear what the inclusion criteria or methods for appraisal are (MacDonald, 2003). In defense of narrative reviews, Collins et al. (2005) note they can have the advantage over systematic reviews with respect to tackling comprehensive topics in a much wider way, while systematic reviews are more favorable for specific questions being answered (Baethge et al., 2019). These authors call for narrative reviews to be strengthened by adopting some of the techniques of systematic reviews, such as 
transparency in reporting methods (Collins et al., 2005). Therefore, the current review used the RE-AIM framework as a means to support transparency. The RE-AIM is an intervention checklist that provides a lens through which to evaluate the quality and impact of an intervention. It allows the researcher to focus on the extent to which the intervention attracts its targeted participants, the improvements or changes in the participants' lives, the setting/site/context of the intervention, its fidelity, transferability, and adaptability, as well as the intervention's evaluation and maintenance (Belza et al., 2006). Moreover, because parents serve as intervention agents (Faith et al., 2012), full reporting of the RE-AIM components can provide insight for the development of the types of parental interventions that are most likely to be adopted and implemented by parents (Schlechter et al., 2016).

\section{Search strategy}

Between the period of August and October 2018, a comprehensive search was conducted by the reviewer in the following eight databases: Springerlink, Health South Consumer Edition, PsycArticles, Academic Search Complete, SocINDEX, Sabinet, and Pubmed. These databases were chosen due to their content matter and accessibility by the primary author. The following keywords were used in the order documented: "Feeding education programs in Africa", "Nutritional programs in Africa", "Parental programs in Africa", "Feeding styles in Africa", "Nutrition education in Africa", and "Nutritional education programs in Africa". The following keywords were then searched in academic journals: "Nutritional interventions in Africa", "Parental nutritional education programs in Africa", "Feeding and parent practice programs", "Parental feeding programs", "Feeding and parent practice interventions", and "Parent feeding program". These keywords were searched for in quotation marks. The Boolean operator 'OR' was used between these phrases. Very few article hits were retrieved at this stage, with only the following countries receiving hits on articles: Angola, Lesotho, Ghana, Mozambique, Zimbabwe, Zambia, Namibia, Kenya, Swaziland, and Uganda. Due to the limited number of articles being retrieved, the search was conducted again with the removal of the word Africa, but the use of all the same search words. All published, English-language, peerreviewed studies within the last ten years (2008-April 2018) were considered for review as the authors wanted to ensure that the information was current and appropriate.

\section{Study selection}

To be eligible to be included in the research, studies had to demonstrate the relevance of the primary caregivers' involvement, as well as meet inclusion criteria. Primacy caregivers' relevance was defined as studies that: (1) included primary caregivers' involvement in interventions to improve child dietary intake; (2) addressed whether primary caregivers' involvement enhances intervention effectiveness; and (3) addressed what type of involvement is most effective in achieving the desired outcome. Studies were included if they: (1) included a control/comparison group; (2) included an intervention focused on primary caregivers' nutrition knowledge improvement, obesity prevention, chronic disease prevention, or health promotion intervention; (3) included a primary caregiver component, defined as an intervention that directly engaged primary caregivers to be intervention agents by supporting or assisting children or adolescents to achieve changes in dietary intake; (4) included child and family dietary intake as a behavior change; (5) recruited primary caregivers with children (3-12 years), or adolescents (13-18 years) and included only humans; (6) published in a peer-reviewed, English-language journal between January 2008 and 2018; (7) included qualitative, quantitative, and mixed-methods studies; and (8) included case studies. Criteria for exclusion were: (1) studies without an intervention component $(n=5325)$; (2) intervention programs designed solely to treat overweight or obese children ( $n=1020)$; (3) programs that enrolled children with a specific medical problem that could impact diet or weight $(n=1065)$; (4) studies for which statistical analysis of outcome data were not reported (e.g., trial protocols, unless complementary to a study that met eligibility criteria) $(n=152)$; (5) literature reviews or commentary $(n=275)$; (6) studies with diet as a correlate and not an outcome $(n=151)$; and (7) program evaluation or pilot studies $(n=175)$. A flow diagram of the search process is depicted in Figure 1: Flow diagram based on the PRISMA statement.

\section{Procedure}

The review was conducted on three levels. The first was to screen the titles of all identified records $(n=8220)$ to assess whether the article was within the parameters of the review. At the second level of review the abstract was further assessed and articles which satisfied all inclusion criteria were deemed eligible for appraisal. Eight thousand one hundred sixty-three $(n=8163)$ articles were deemed unsuitable due to the following reasons: (1) the studies were out of the scope of the review (focusing on breastfeeding, first thousand days of life, or HIV or specific disease focus, etc.); (2) articles were not published in English; (3) articles required payment and were not freely available; and (4) studies were grey literature, systematic reviews, or conceptual papers only. Duplicate articles across the different databases were deleted $(n=12)$. Forty-six $(n=46)$ of the retrieved articles' interventions were deemed eligible and 42 $(n=42)$ were excluded based on the RE-AIM approach with a series of "yes" or "no" questions. In the end, a total of four articles $(n=4)$ were included in the study.

\section{Using the RE-AIM framework}

The RE-AIM framework was used to allow for crosscomparison of core components inherent in health 


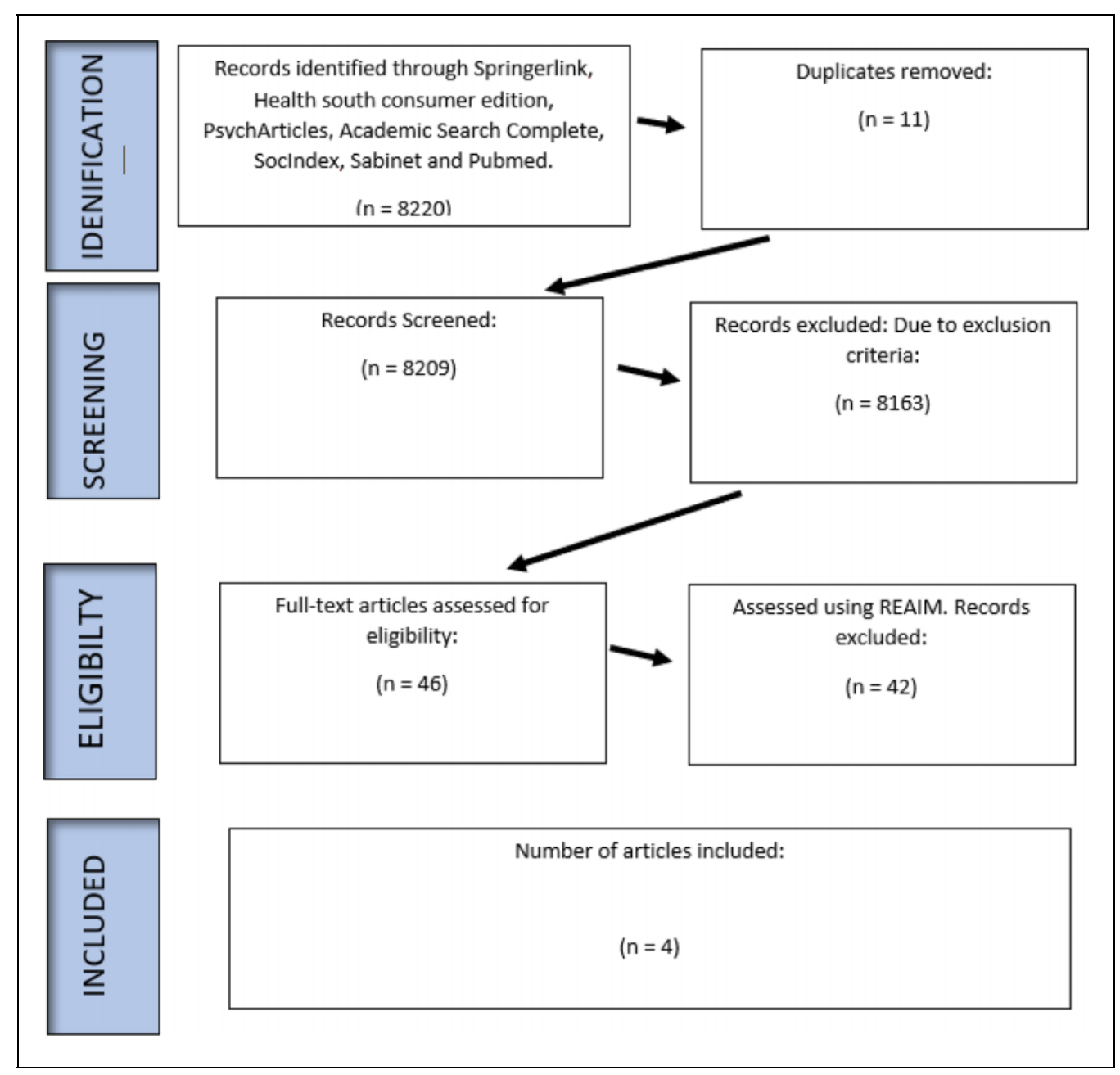

Figure I. Flow diagram of identified records.

promotion interventions. The framework is widely used to facilitate the development, delivery, and evaluation of health interventions according to five elements, namely: (1) Reach - refers to which target population the intervention is intended to reach as well as if the intervention was used on the intended target population; (2) Effectiveness - refers to the intervention achieving the objectives/outcomes; (3) Adoption - refers to target staff or organization that would adopt the intervention; (4) Implementation - refers to consistency and adaption of intervention protocol to practice; (5) Maintenance - refers to intervention effects on participants over a period of time (Matthews et al., 2014). All three levels of the review were performed by both the primary researcher as well as a second reviewer. Data extraction according to these elements is shown in Table 1.

\section{Results}

The results of the search strategy did not yield any studies that answered the study selection criteria of being specific to Africa. It yielded four studies that met the selection criteria but focused outside of Africa. These studies are assessed according to the RE-AIM framework below.

\section{Reach}

The interventions recruited parents via day care centers, preschools, and family day cares that dealt directly with parents. In some studies recruitment was done by the actual facility staff who ran the facilities or playgrounds, as well as by early childhood health professionals who have contact with parents of children in the target group (Duncanson et al., 2012; Elinder et al., 2018; Morgan et al., 2011; Skouteris et al., 2010). Parents of children who were aged $2-4$ were recruited to be part of one of the four studies (Skouteris et al., 2010). Others were recruited by the school nurse or directly by the research team during school term meetings (Elinder et al., 2018). One study specifically focused on overweight and obese fathers (Morgan et al., 2011). Overweight or obese (BMI between 25 and $40 \mathrm{~kg} / \mathrm{m}^{2}$ ) fathers (aged 18-65 years) with a child aged 5-12 years (i.e., primary school age) were recruited from the local communities using the following strategies: local media releases (print, TV, and radio), school newsletters items, school-based presentations, fliers distributed through community notice boards and local businesses, paid advertisements in local newspapers, local networks of sports clubs, service clubs, local government, 


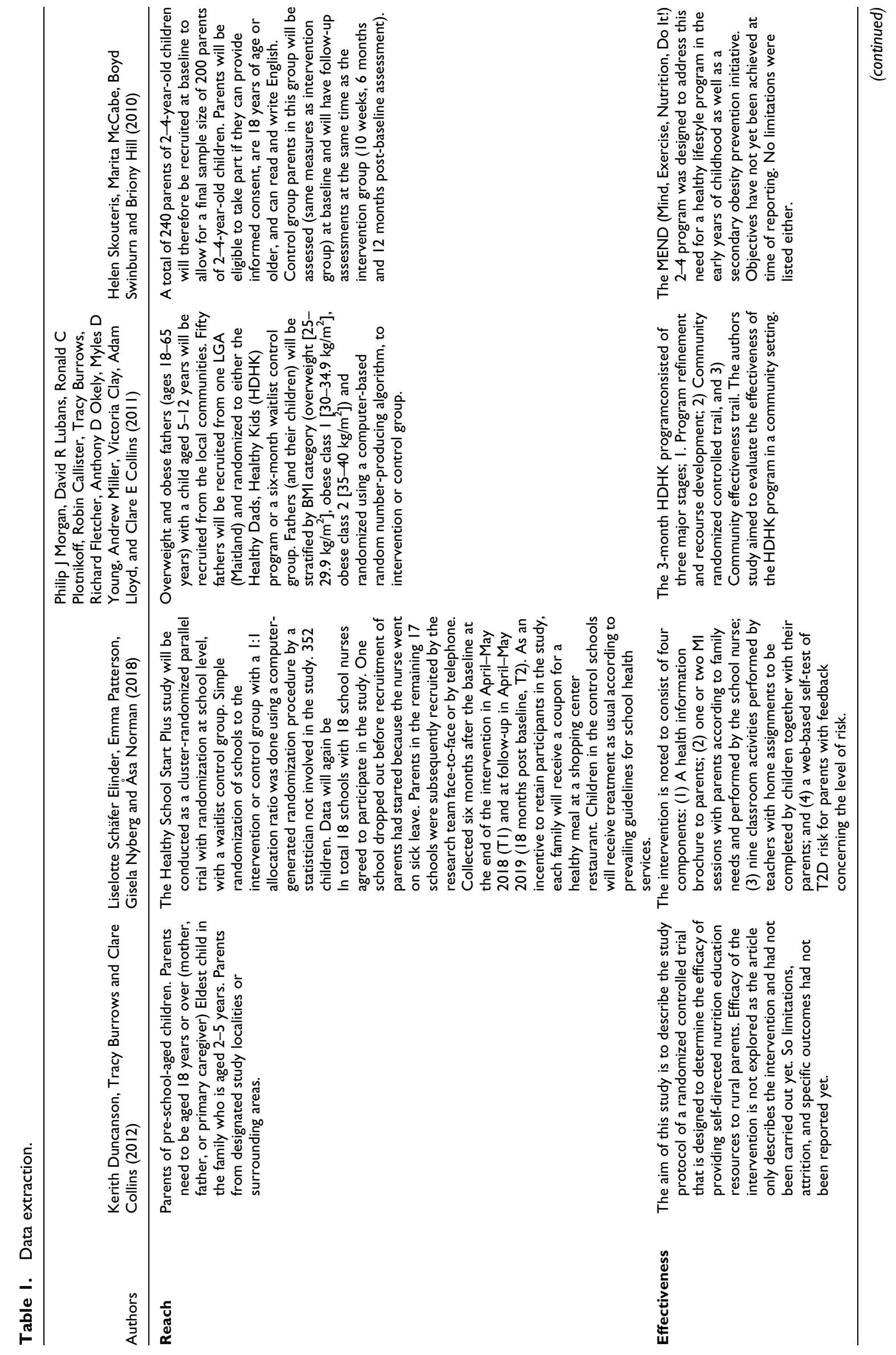




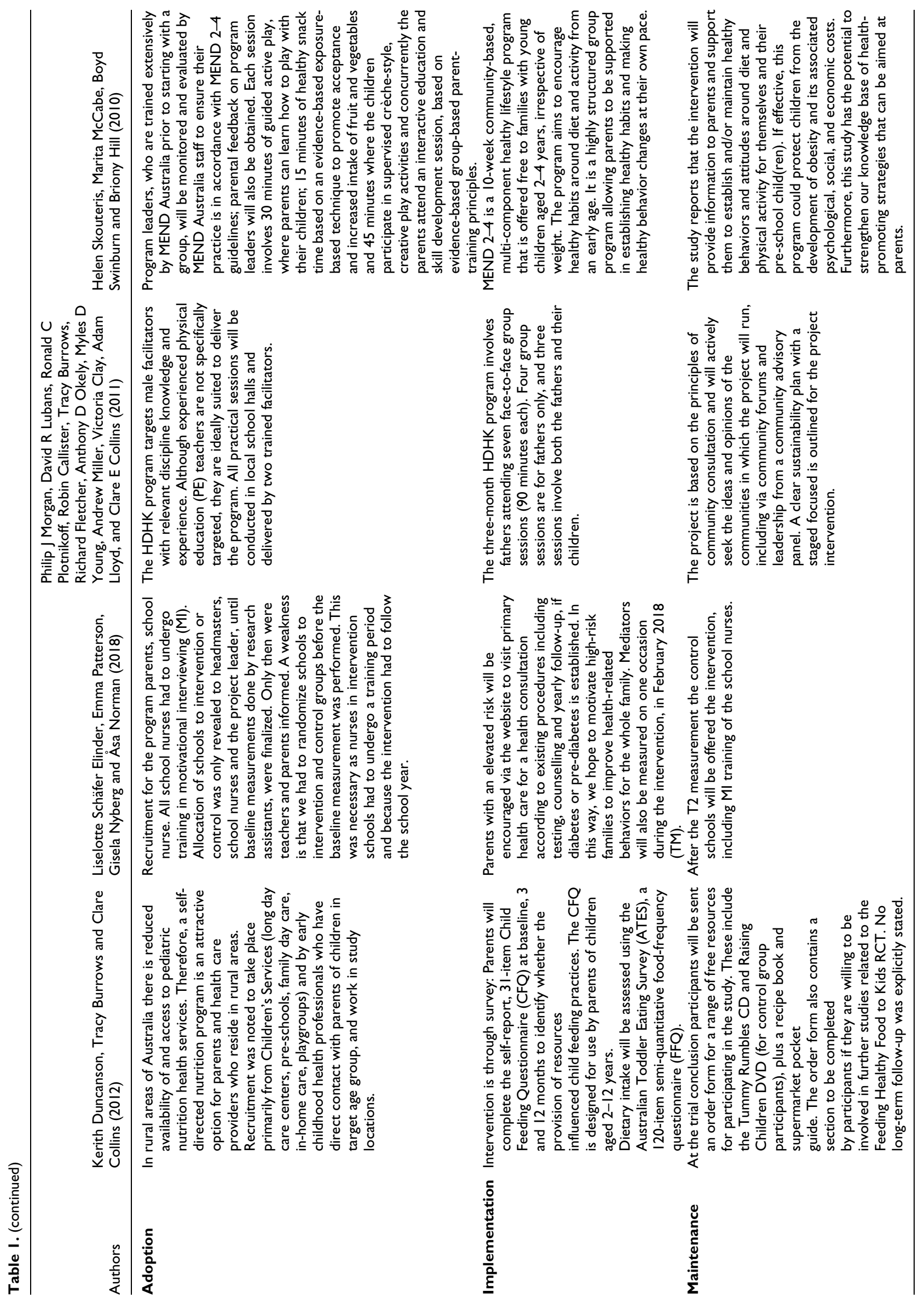


and community health facilities (Morgan et al., 2011). Once selected fathers came forward, they were then screened for eligibility via telephone. Duncanson et al. (2012) also used a focused recruitment strategy disseminating flyers and newspaper advertisements in order to maximize recruitment. The success rate of various strategies was not provided as the articles reviewed only focused on the intervention and not on implementation.

\section{Effectiveness}

The reviewed studies each had a clear aim and purpose. The Duncanson et al. (2012) study described the study protocol of a randomized controlled trial that was designed to determine the efficacy of providing self-directed nutrition education resources to rural parents. This would be achieved by measuring the impact of providing parents of pre-school-aged children with self-directed nutrition and parenting resources on selected dietary and child feeding factor variables, following intention-to-treat principles, with secondary per-protocol analysis (Duncanson et al., 2012). All the interventions had clear inclusion and exclusion criteria and aimed for more than 100 participants. Participants were required to be fathers or parents older than 18 years, who have children within the required age range of the study (Duncanson et al., 2012; Elinder et al., 2018; Morgan et al., 2011; Skouteris et al., 2010). Duncanson et al. (2012) provided a clear description of the theoretical framework for the study and placed focus on the aspect that changing the dietary intake of children requires a change in the feeding practices of parents. Therefore, the Theory of Planned Behavior (TPB) could be used for effective change due to the fact that it was originally developed to predict human behavior change. The Elinder et al. (2010) Healthy School Start Plus study's purpose was to compare the effect of the program to standard care outcomes related to diet, physical activity, and weight development of children. Morgan et al.'s (2011) Healthy Dads, Healthy Kids (HDHK) study is a trial that consists of three stages: Stage 1 - Program refinement and resource development; Stage 2 - Community randomized controlled trial (RCT); and Stage 3 - Community effectiveness trial. The focus of the trial was on areas in Australia which are considered to be involved in the mining manufacturing industries. Fifty-two percent (52\%) of men in Australia who work in the mining industry are engaged in shift work, which is associated with increased health issues compared with working daytime hours, and is an independent predictor of increased body mass index (Morgan et al., 2011). The Mind Exercise Nutrition Do It! (MEND) program aims to encourage healthy habits around diet and activity from an early age. It is a highly structured group program allowing parents to be supported in establishing healthy habits and making healthy behavior changes at their own pace (Skouteris et al., 2010).

\section{Adoption}

Most of the interventions made use of trained facilitators (Elinder et al., 2018; Morgan et al., 2011). The HDHK program targeted male facilitators with relevant discipline knowledge and experience, which allowed for an easier process of engaging with fathers (Morgan et al., 2011). MEND used 2-4 leaders that were provided with comprehensive training by MEND Australia. These participants received a leader's manual together with a full resource kit (Skouteris et al., 2010). One intervention provided a resources folder to participants and would follow up with participants through telephonic reminders; this meant the intervention was home-based or wherever the participant was able to review the resource material (Duncanson et al., 2012).

\section{Implementation}

The intervention layouts varied across studies with either face-to-face workshops provided by the school nurse, prepared material (CD, electronic, resource file) that needed to be reviewed by the parents, or the use of a webbased testing tool (Skouteris et al., 2010; Duncanson et al., 2012; Elinder et al., 2018; Morgan et al., 2011). Each study had a number of face-to-face sessions as outlined: The three-month HDHK program involves fathers attending seven face-to-face group sessions (90 minutes each), four group sessions are for fathers only, and three sessions involve both the fathers and their children (Morgan et al., 2011). The MEND 2-4 program involves 10 weekly 90minute workshops relating to general nutrition, physical activity, and behaviors that are typically held at community health and maternal and child health centers, where parents and their pre-school-aged children attend together (Skouteris et al., 2010). Each program group consisted of 6-10 child-parent dyads and a MEND 2-4 trained program leader. It included classroom activities and home assignments. Nine manual-based classroom sessions of approximately 30 minutes in duration were delivered by the teacher. Various pedagogic materials were provided for the sessions, including a workbook for the children. In order to retain families in the study and to achieve a high level of adherence to the intervention components, two of the four studies implemented e-mails, calls, and text messages to parents and participants (Elinder et al., 2018; Morgan et al., 2011).

\section{Maintenance}

All four studies reported that the intervention would be followed up. This would occur through direct calls with participants where an offering of resources would be made available online (Duncanson et al., 2012). This would allow parents to have access to up-to-date research on child nutritional needs or parent feeding. One study stated that after the end of the intervention, semi-structured interviews 
with parents, school nurses, and head teachers would be performed to explore their views on the acceptability and feasibility of the intervention (Elinder et al., 2018). However, the studies did not indicate when the interviews would be conducted after the completion of the intervention. The HDHK project was based on the principles of community consultation and actively sought the ideas and opinions of the communities in which the project ran and in some cases was funded as well. Community advisory panel members represented a broad range of areas including education, local government, health, sports groups, and service clubs (Morgan et al., 2011). This community forums approach allowed for maintenance of the program as a whole.

\section{Discussion}

This review paper aimed to synthesize recent research evidence to identify and describe best practice models, programs, or processes in primary caregivers' nutritional and feeding intervention development in Africa. This was done through a narrative review. No studies that were conducted in Africa met the inclusion criteria; therefore, studies that met all the other criteria were selected.

The interventions reviewed provide a varied approach to implementation, focusing on the interventions being provided by trained facilitators to a specific target audience, while being conducted either face-to-face in workshops or with the use of online resources (Skouteris et al., 2010; Duncanson et al., 2012; Elinder et al., 2018; Morgan et al., 2011). In the review, only one article focused on the enablers, barriers, and resources with respect to engaging health behavior change in primary caregivers and families as a whole (Duncanson et al., 2012). This is a limitation of the other reviewed articles, as in order to develop similar interventions, it is necessary to have a grasp on the enablers and barriers involved, to increase positive uptake of the intervention and determine its sustainability. A strength of each article reviewed is that they clearly focus their interventions on providing information to primary caregivers. This information is intended to provide support to the caregivers with respect to maintenance, as well as in some cases establishing health behaviors and attitudes to diet and physical activity for both themselves and children. KiefnerBurmeister et al. (2016) state that the future health of children is based, in part, on the behaviors that parents engage in. Therefore, parental education on healthy feeding behaviors and parenting styles may contribute to the health of future generations.

The said behavior change needs to be mediated through the development of implementation interventions which draw on theory, evidence, and, in some cases, practical issues. Theory can be used to understand the factors that might influence the clinical behavior change which is being targeted, as well as to underpin possible techniques that could be used to change positive and negative health behaviors (Michie et al., 2005). Theory was clearly expressed for the formulation of interventions in three of the four reviewed articles, such as the theory of planned behavior (TPB), social cognitive theory (SCT), family systems theory (FST), as well as systematic reviews forming the basis of different intervention idea groupings. Duncanson et al. (2012) state that changing the dietary intake of children requires change to the feeding practices of parents as well as through the use of TPB. The key components of the TPB are proposed to predict the child feeding practices or behaviors of parents. TPB was originally developed to predict and explain human social behavior and is being used in the Feeding Healthy Food to Kids program to serve as a framework for behavior change interventions. The HDHK program was envisioned to help fathers achieve their weight loss goals, become healthy role models, and promote healthy behaviors for their children. The HDHK is based on both FST and SCT, while the Health School Start Plus (Elinder et al., 2018) is only based on the latter. The SCT constructs should be targeted and operationalized: self-efficacy, goals/intention, outcome expectations, perceived facilitators and barriers to change, and social support (Morgan et al., 2011). FST postulates a complex theoretical framework of reciprocal relationships among family members. That is, when a parent changes his or her physical activity and dietary behaviors, this will be reflected in the child's behavior (Morgan et al., 2011). This is a weakness in the Skouteris et al. (2010) study, which does not provide the reader with a clear basis of intervention theory.

The HDHK intervention (Morgan et al., 2011) was the only one of the four articles reviewed that outlined both primary and secondary outcomes of the intervention. The primary intervention involved recording the intake at home of a composite score of indicator foods of importance for energy balance and health, namely fruit and vegetables (healthy foods), unhealthy foods (sweets, ice cream, buns/ cakes, crisps), and unhealthy drinks high in added sugar (sugar-sweetened beverages) (Morgan et al., 2011). Previous studies have found that the use of indicator food in comparable population samples showed that diet was a discriminating factor between different socioeconomic and ethnic groups and also improved as a result of the intervention (Nyberg et al., 2016). This would make the HDHK intervention more appropriate for low socioeconomic environments. Those designing interventions in low socioeconomic communities will need to give considerable thought to the affordability and accessibility of their interventions, especially with the use of CDs and electronic sessions for implementation, since lower socioeconomic communities will have less access to computers. However, in comparison with web-based interactive interventions, they have been found to offer personalized advice, education, and feedback messages about health behavior. They also served as attractive features for people to continue using the websites long after the official program has ended (Maon et al., 2012). A preferred consideration for intervention developers in selected studies was using local facilitators, e.g., school nurses, community 
workers, or peers of potential participants (Duncanson et al., 2012; Elinder et al., 2018; Nyberg et al., 2016).

Two studies highlighted ways they would fill their research gaps. Feeding Healthy Food to Kids stated that it would address issues by analyzing the dietary intake of 2 5-year-old children, using a validated food frequency questionnaire completed by parent proxy. As such, this study provided additional insight into the potential for a parent-focused nutrition intervention to influence the dietary intake of rural pre-school-aged children (Duncanson et al., 2012). The Healthy School Start Plus intervention stated that it was a multi-disciplinary theory-based program which would fill a large knowledge gap regarding evidence-based practice within school health services to promote health-related behaviors and prevent overweight and obesity in children. The program was designed to be fully integrated into normal school routines, making it highly sustainable once the necessary support from the school management is secured (Elinder et al., 2018). Some weaknesses from the study design though are that the primary outcome (dietary intake of indicator foods in the home environment) is based on self-reporting, as are the theory-based mediators (Elinder et al., 2018). In selfreporting, individuals could fail to disclose the true portions consumed. The article did mention that measures to mitigate this include the use of photos to validate the estimated selected food items and quantity that children were consuming. This makes this intervention a costeffective approach that can be adaptable in many settings, and easily replicated.

\section{Recommendations}

None of the reviewed interventions were evaluated and therefore no information is available on the interventions' uptake. It is recommended that follow-up articles are conducted to analyze the outcomes over a period. If this review is a microcosm of the larger society, it can be premised that not only is clinical and academic input needed to develop more nutrition interventions and programs in Africa, and on other continents, but that political and policy influence is also required to provide the funding mechanisms needed for implementation and sustainability of these interventions. It is recommended that further investigation is conducted, using other search criteria, possibly using double-duty actions for malnutrition in Africa, as these may yield more studies that are focused on primary caregivers' development of nutritional and feeding interventions.

\section{Limitations}

A limitation of the research article is that the search criteria was initially focused on Africa. Unfortunately, there were no studies that met the inclusion criteria for Africa, and, therefore, the inclusion criteria were amended to include all countries. The interventions reviewed were limiting as they only focused on the intervention stage and not on implementation or evaluation. However, the inclusion criteria could be too strict, or they would not have yielded any results at all. A narrative review was used for this study due to the limited number of published articles found on the topic and therefore a systematic review was not achievable. Narrative overviews are effective in that they allow you to easily synthesize information to keep up to date, receive continuing education credits, or challenge your way of thinking. However, there is a belief they are not a form of evidence that should be used frequently when making decisions regarding how to solve specific clinical patient problems.

\section{Declaration of conflicting interests}

The authors have no conflicts of interest to declare.

\section{Ethics}

Ethical clearance was received for this study from the University of the Western Cape, Senate ethics committee with clearance number 14/10/34.

\section{Funding}

The authors disclosed receipt of the following financial support for the research, authorship, and/or publication of this article: This work was supported by the National Research Foundation (NRF) South Africa (grant number 110805).

\section{ORCID iD}

Melissa Brown (D) https://orcid.org/0000-0002-0375-4057

\section{References}

Ahishakiye J, Bouwman L, Brouwer ID, et al. (2019) Challenges and responses to infant and young child feeding in rural Rwanda: A qualitative study. Journal of Health, Population and Nutrition 38(1): 43.

Baethge C, Goldbeck-Wood S and Mertens S (2019) SANRA-a scale for the quality assessment of narrative review articles. Research Integrity and Peer Review 4(1): 1-7.

Belza B, Toobert D and Glasgow R (2006) RE-AIM for program planning: Overview and applications. The National Council on Aging's Center for Healthy Aging 2019: 1-16. Available at: http://www.re-aim.org/2007-re-aim-for-program-planningand-evaluation-overview-and-applications/ (accessed on the 17th July 2019).

Bhutta ZA (2017) Global child nutrition and the Sustainable Development Goals. The Lancet Child and Adolescent Health 1(4): 256-257.

Cirak O (2018) Nutritional factors in etiology of childhood obesity. Gen Med Open 4(4): 1-5.

Cluss PA, Ewing L, King WC, et al. (2013) Nutrition knowledge of low-income parents of obese children. Translational Behavioral Medicine 3(2): 218-225.

Collins LM, Murphy SA, Nair VN, et al. (2005) A strategy for optimizing and evaluating behavioral interventions. Annals of Behavioral Medicine 30(1): 65-73.

Craigie AM, Lake AA, Kelly SA, et al. (2011) Tracking of obesity-related behaviours from childhood to adulthood: A systematic review. Maturitas 70(3): 266-284. 
Development Initiatives (2018) Global Nutrition Report: Shining a light to spur action on nutrition. Bristol, UK: Development Initiatives.

Duncanson K, Burrows T and Collins C (2012) Study protocol of a parent-focused child feeding and dietary intake intervention: The feeding healthy food to kids randomised controlled trial. BMC Public Health 12(1): 564.

Elinder LS, Patterson E, Nyberg G, et al. (2018) A Healthy School Start Plus for prevention of childhood overweight and obesity in disadvantaged areas through parental support in the school setting - study protocol for a parallel group cluster randomised trial. BMC Public Health 18(1): 459.

Faith SM, Linda VH, Appel LJ, et al. (2012) Evaluating parents and adult caregivers as "agents of change" for treating obese children: evidence for parent behavior change strategies and research gaps: a scientific statement from the American Heart Association. Circulation 125(9): 1186-1207. DOI: 10.1161/ CIR.0b013e31824607ee.

undefined

Galloway AT, Fiorito LM, Francis LA, et al. (2006) 'Finish your soup': Counterproductive effects of pressuring children to eat on intake and affect. Appetite 46(3): 318-323.

Golan M and Weizman A (2001) Familial approach to the treatment of childhood obesity: Conceptual model. Journal of Nutrition Education 33(2): 102-107.

Green BN, Johnson CD and Adams A (2006) Writing narrative literature reviews for peer-reviewed journals: Secrets of the trade. Journal of Chiropractic Medicine 5(3): 101-117.

Hayward J, Jacka FN, Skouteris H, et al. (2016) Lifestyle factors and adolescent depressive symptomatology: Associations and effect sizes of diet, physical activity and sedentary behaviour. The Australian and New Zealand Journal of Psychiatry 50(11): 1064-1073.

Hingle MD, O'Connor TM, Dave JM, et al. (2010) Parental involvement in interventions to improve child dietary intake: A systematic review. Preventive Medicine 51(2): 103-111.

Hong SA and Peltzer K (2017) Dietary behaviour, psychological well-being and mental distress among adolescents in Korea. Child and Adolescent Psychiatry and Mental Health 11: 56.

Jahan N, Naveed S, Zeshan M, et al. (2016) How to conduct a systematic review: A narrative literature review. Cureus 8(11): e864-e864.

Kelder SH, Perry CL, Klepp KI, et al. (1994) Longitudinal tracking of adolescent smoking, physical activity, and food choice behaviors. American Journal of Public Health 84(7): 1121-1126.

Kiefner-Burmeister A, Hoffmann D, Zbur S, et al. (2016) Implementation of parental feeding practices: Does parenting style matter? Public Health Nutrition 19(13): 2410-2414.

MacDonald GM (2003) Using Systematic Reviews to Improve Social Care. London: Social Care Institute for Excellence.

Maon S, Edirippulige S, Ware R, et al. (2012) The use of webbased interventions to prevent excessive weight gain. Journal of Telemedicine and Telecare 18(1): 37-41.

Matthews L, Kirk A, Macmillan F, et al. (2014) Can physical activity interventions for adults with type 2 diabetes be translated into practice settings? A systematic review using the RE-
AIM framework. Translational Behavioral Medicine 4(1): 60-78.

Michie S, Johnston M, Abraham C, et al. (2005) Making psychological theory useful for implementing evidence based practice: A consensus approach. Quality and Safety in Health Care 2005: 26-33.

Mills PR, Weidmann AE and Stewart D (2016) Hospital discharge information communication and prescribing errors: A narrative literature overview. European Journal of Hospital Pharmacy 23(1): 3-10.

Moghames P, Hammami N, Hwalla N, et al. (2016) Validity and reliability of a food frequency questionnaire to estimate dietary intake among Lebanese children. Nutrition Journal 15(1): 4.

Morgan PJ, Lubans DR, Plotnikoff RC, et al. (2011) The 'Healthy Dads, Healthy Kids' community effectiveness trial: Study protocol of a community-based healthy lifestyle program for fathers and their children. BMC Public Health 11(1): 876.

Nyberg G, Norman $\AA$, Sundblom E, et al. (2016) Effectiveness of a universal parental support programme to promote health behaviors and prevent overweight and obesity in 6-year-old children in disadvantaged areas, the Healthy School Start Study II, a cluster-randomised controlled trial. International Journal of Behavioral Nutrition and Physical Activity 13(1): 4.

Patel C, Karasouli E, Shuttlewood E, et al. (2018) Food parenting practices among parents with overweight and obesity: A systematic review. Nutrients 10(12): 1966.

Romanos-Nanclares A, Zazpe I, Santiago S, et al. (2018) Influence of parental healthy-eating attitudes and nutritional knowledge on nutritional adequacy and diet quality among preschoolers: The SENDO Project. Nutrients 10(12): 1875.

Salk RH, Hyde JS and Abramson LY (2017) Gender differences in depression in representative national samples: Metaanalyses of diagnoses and symptoms. Psychological Bulletin 143(8): 783-822.

Sanders D and Reynolds L (2017) Ending stunting: Transforming the health system so children can thrive. In: Children's Institute U of CT (ed) South African Child Gauge. Cape Town: Jamieson L, pp. 68-76.

Schlechter CR, Rosenkranz RR, Guagliano JM, et al. (2016) A systematic review of children's dietary interventions with parents as change agents: Application of the RE-AIM framework. Preventive Medicine 91: 233-243.

Shloim N, Edelson LR, Martin N, et al. (2015) Parenting styles, feeding styles, feeding practices, and weight status in 4-12 year-old children: A systematic review of the literature. Frontiers in Psychology. Available at: https://www.frontiersin.org/ article/10.3389/fpsyg.2015.01849 (accessed 10th October 2020).

Skouteris H, McCabe M, Swinburn B, et al. (2010) Healthy eating and obesity prevention for preschoolers: A randomised controlled trial. BMC Public Health 10: 220.

Walsh F (2003) Family resilience: A framework for clinical practice. Family Process 42(1): 1-18.

Yavorsky JE, Dush CMK and Schoppe-Sullivan SJ (2015) The production of inequality: The gender division of labor across the transition to parenthood. Journal of Marriage and the Family 77(3): 662-679. 